\title{
PAF1-complex-mediated histone methylation of FLOWERING LOCUS $C$ chromatin is required for the vernalization-responsive, winter-annual habit in Arabidopsis
}

\author{
Yuehui He, ${ }^{1,3}$ Mark R. Doyle, ${ }^{1,2,3}$ and Richard M. Amasino ${ }^{1,2,4}$ \\ ${ }^{1}$ Department of Biochemistry and ${ }^{2}$ Program in Cellular and Molecular Biology, University of Wisconsin, \\ Madison, Wisconsin 53706, USA
}

\begin{abstract}
The winter-annual habit (which typically involves a requirement for exposure to the cold of winter to flower in the spring) in Arabidopsis thaliana is mainly due to the repression of flowering by relatively high levels of $F L C$ expression. Exposure to prolonged cold attenuates $F L C$ expression through a process known as vernalization and thus permits flowering to occur in the spring. Here we show that the elevated FLC expression characteristic of nonvernalized winter annuals requires two genes, EARLY FLOWERING 7 (ELF7) and EARLY FLOWERING 8 (ELF8), that are homologs of components of the PAF1 complex of Saccharomyces cerevisiae. Furthermore, ELF7 and ELF8 are also required for the expression of other genes in the FLC clade of flowering repressors such as MAF2 and FLM. FLC, FLM, and MAF2 are involved in multiple flowering pathways that account for the broad effects of elf7 and elf8 mutations on flowering behavior. ELF7 and ELF8 are required for the enhancement of histone 3 trimethylation at Lys 4 in FLC chromatin. This modification of FLC chromatin appears to be required to elevate $F L C$ expression to levels that can delay flowering in plants that have not been vernalized. A model of the role of $E L F 7, E L F 8$, and other previously described genes in the modification of the chromatin of flowering repressors is presented.
\end{abstract}

[Keywords: FLC; PAF1 complex; EARLY FLOWERING 7; EARLY FLOWERING 8; MADS-box genes; histone methylation]

Supplemental material is available at http://www.genesdev.org.

Received July 28, 2004; revised version accepted September 17, 2004.

The floral transition is a major developmental switch in the plant life cycle that must be properly timed to ensure maximal reproductive success. This transition involves coordinating responses to environmental cues such as day length, light quality, and temperature with endogenous factors such as developmental status and age. In Arabidopsis thaliana, a facultative long-day plant, flowering time is regulated by five distinct, yet linked pathways (Boss et al. 2004; Putterill et al. 2004). The photoperiod and vernalization pathways accelerate flowering in response to day length and long-term cold exposure, respectively. A thermosensory pathway promotes flowering in response to increased ambient temperature (Blazquez et al. 2003). The autonomous pathway and a

\footnotetext{
${ }^{3}$ These authors contributed equally to this work.

${ }^{4}$ Corresponding author.

E-MAIL amasino@biochem.wisc.edu; FAX (608) 262-3453.

Article published online ahead of print. Article and publication date are

at http://www.genesdev.org/cgi/doi/10.1101/gad.1244504.
}

gibberellin-dependent pathway promote flowering largely in response to developmental signals. Outputs from these pathways are integrated by a set of common downstream target genes, "flowering-time integrators"; expression of these integrators leads to the induction of floral-meristem-identity genes and thus flowering (Boss et al. 2004; Putterill et al. 2004).

Flowering-time variation exists among Arabidopsis accessions. The genetic difference between a winter-annual habit (late flowering without vernalization) and a rapid-flowering habit (rapid flowering without vernalization) is often determined by allelic variation at FRIGIDA (FRI) or FLOWERING LOCUS C (FLC) (Burn et al. 1993; Lee et al. 1993, 1994; Clarke and Dean 1994; Koornneef et al. 1994). FLC is a MADS-box transcriptional regulator (Michaels and Amasino 1999; Sheldon et al. 1999) that inhibits the floral transition largely by reducing the expression of flowering-time integrators such as SUPPRESSOR OF OVEREXPRESSION OF CONSTANS 1 and FT (Hepworth et al. 2002). The role of FRI is to up-regulate 
Table 1. Primary leaf number at bolting of elf7 mutants

\begin{tabular}{|c|c|c|c|c|c|}
\hline Day length & Ws & elf7-1 & Col & elf7-2 & elf7-3 \\
\hline Short days & $\begin{array}{c}36.9 \pm 4.4 \\
(7.3 \pm 0.8)\end{array}$ & $\begin{array}{c}14.4 \pm 1.4 \\
(5.1 \pm 0.6)\end{array}$ & $\begin{array}{c}66.9 \pm 3.9 \\
(10.7 \pm 1.5)\end{array}$ & $\begin{array}{c}19.2 \pm 2.6 \\
(4.1 \pm 0.6)\end{array}$ & $\begin{aligned} 21.2 & \pm 2.9 \\
5.6 & \pm 0.8\end{aligned}$ \\
\hline Long days & $\begin{array}{c}9.9 \pm 1.1 \\
(2.9 \pm 0.4)\end{array}$ & $\begin{array}{c}6.8 \pm 0.7 \\
(3.4 \pm 0.5)\end{array}$ & $\begin{array}{c}12.2 \pm 1.2 \\
(3.2 \pm 0.7)\end{array}$ & $\begin{array}{c}6.6 \pm 0.7 \\
(2.8 \pm 0.7)\end{array}$ & $\begin{array}{l}\mathrm{ND}^{\mathrm{a}} \\
\mathrm{ND}^{\mathrm{a}}\end{array}$ \\
\hline
\end{tabular}

Values shown are mean number \pm standard deviation of rosette and cauline leaves (in parentheses); 10 plants were scored for each line. ${ }^{a} \mathrm{ND}$, not determined.

FLC expression to levels that inhibit flowering (Michaels and Amasino 1999; Sheldon et al. 1999). Winter annuals have dominant alleles of FRI and FLC, whereas many rapid-flowering accessions have a nonfunctional fri allele (Johanson et al. 2000) or an allele of FLC that is not up-regulated by FRI (Gazzani et al. 2003; Michaels et al. 2003).

Autonomous-pathway mutations delay the onset of flowering in rapid-flowering accessions (Boss et al. 2004). In fact, such mutations cause otherwise rapid-flowering accessions to behave like FRI-containing winter annuals. Both FRI and mutations in autonomous-pathway genes result in increased FLC expression, and loss-of-function flc mutations suppress the delayed flowering of FRI-containing lines or autonomous-pathway mutants (Michaels and Amasino 2001). Thus, the normal role of autonomous-pathway genes is to repress FLC, and FRI can overcome this suppression (Michaels and Amasino 2001). The increased FLC expression and delayed flowering in both FRI and autonomous-pathway mutants can be reversed by exposure to the prolonged cold typical of a winter season by a process known as vernalization (Michaels and Amasino 2000; Boss et al. 2004).

Recent studies have begun to reveal the molecular mechanisms involved in FLC repression. Two genes in the autonomous pathway, FLOWERING LOCUS D $(F L D)$ and $F V E$, encode proteins involved in deacetylating core histone tails of FLC chromatin (He et al. 2003; Ausin et al. 2004). Vernalization leads to repressive histone modifications in FLC chromatin including deacetylation, and increased methylation of Lys 9 and Lys 27 of histone 3 (Bastow et al. 2004; Sung and Amasino 2004). These modifications are characteristic of a stable heterochromatin conformation.

Given the central role of $F L C$ in controlling flowering time, understanding its regulation at a molecular level is of great interest. Genetic analyses have revealed many of the components involved in the regulatory network governing FLC expression. The study of mutants that render a FRI-containing winter-annual line early flowering has led to the identification of several loci that are required for FLC expression: VERNALIZATION INDEPENDENCE 4 (VIP4) (Zhang and van Nocker 2002), VIP3 (Zhang et al. 2003), and PHOTOPERIOD INDEPENDENT EARLY FLOWERING 1 (PIE1) (Noh and Amasino 2003). Also, mutations in EARLY IN SHORT DAYS 4 (ESD4) cause a modest decrease in FLC levels (Reeves et al. 2002; Murtas et al. 2003). However, the molecular mechanism by which these activators regulate FLC expression is not known.
In this report, we present the identification of two genes involved in FLC activation: ELF7, which encodes the Arabidopsis relative of yeast RNA polymerase II (Pol II) Associated Factor 1 (PAF1), and ELF8, which encodes the Arabidopsis relative of yeast CLN Three Requiring 9 (CTR9). Lesions in ELF7 and ELF8 cause early flowering in an otherwise late-flowering, FRI-containing line by suppressing FLC expression, and also cause early flowering in noninductive photoperiods by suppressing expression of the MADS-box genes FLOWERING LOCUS $M$ (FLM) and MADS AFFECTING FLOWERING 2 (MAF2). The expression of these MADS-box flowering repressors is associated with increased histone 3 trimethylation at Lys 4, and this chromatin modification requires ELF7 and ELF8.

\section{Results}

\section{Mutations in ELF7 result in early flowering}

We screened a T-DNA mutagenized population in the Wassilewskija (Ws) accession of Arabidopsis for mutants that flowered more rapidly than wild type in noninductive short days (SD). One mutant, referred to as early-
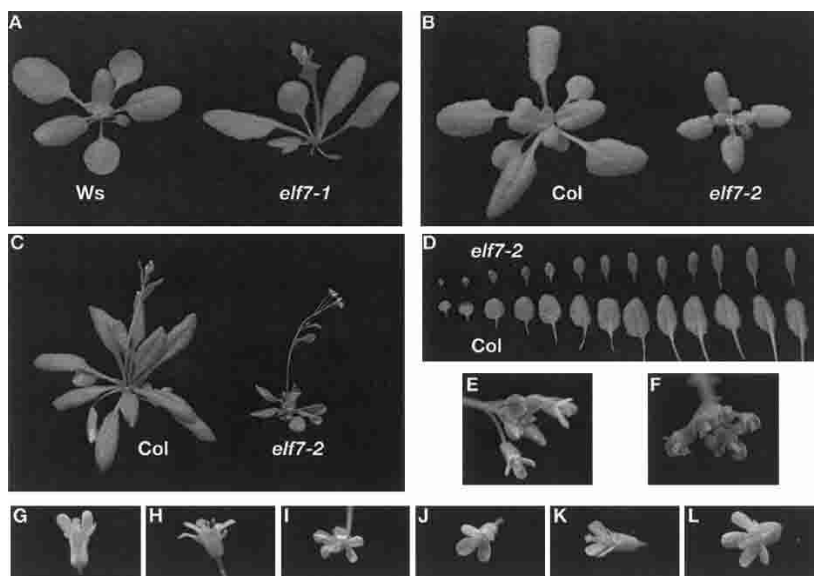

Figure 1. elf7 mutants are early flowering and display additional accession-dependent phenotypes. $(A-C)$ elf7 mutants grown under LD. (D) The first 13 rosette leaves formed on representative elf7-2 and Col plants grown in SD. $(E)$ Flower buds in Col. $(F)$ Flower buds in elf7-2. $(G-L)$ Representative flowers from elf7 and wild types: $\operatorname{Col}(G)$, elf7-2 $(H, I)$, Ws $(J)$, elf7-1 $(K, L)$. elf7-1 and elf7-2 display altered petal number in a fraction of flowers $(I, J)$; in addition, in elf7-2 flowers $(F)$, but not in elf 7-1 flowers, the sepals do not enclose the flower buds. 
He et al.

flowering 7 (elf7), flowered earlier than wild type in both SD and also in inductive long days (LD) (Table 1; Fig. 1A). A backcross to wild type revealed that the elf7 phenotype was recessive. Analysis of the $\mathrm{F}_{2}$ population indicated that the elf7 phenotype segregated as a single Mendelian trait, and that the elf7 line contained a single TDNA locus that cosegregated with the mutant phenotype (data not shown). The sequence of a genomic fragment flanking the left border of the T-DNA indicated that the T-DNA was inserted in the 5'-UTR of At1g79730 (http://www.arabidopsis.org; Fig. 2A).

To confirm that the early-flowering phenotype of elf7 is due to a lesion in At1g79730, two additional T-DNA insertional alleles, elf7-2 and elf7-3, were identified in the Columbia (Col) genetic background from the SALK T-DNA collection (Alonso et al. 2003). Both elf7-2 and elf7-3 flowered earlier than wild-type Col (Table 1). Furthermore, when elf7-1 and elf7-3 were crossed to elf7-2, the $\mathrm{F}_{1}$ progeny flowered early in both SD and LD /data not shown). Finally, a 4.3-kb genomic fragment containing the $A t 1 g 79730$ coding region plus $1.0 \mathrm{~kb}$ of promoter sequence was introduced into elf7-2. This fragment was able to rescue the mutant phenotype (data not shown).

In addition to early flowering, elf7 mutants display other phenotypes, some of which are only found in the Col genetic background. One Col-specific phenotype is that rosette leaves of elf7-2 and elf7-3 are smaller and slightly more curled than wild type (Fig. 1B-D; elf7-2 is shown). There was no difference in the leaves of elf7-1 compared to wild-type Ws (Fig. 1A). Another Col-specific phenotype is that the sepals often fail to enclose flower buds in the latest stages of development (Fig. 1F). The phenotypic difference between elf7 in Col and Ws was analyzed genetically. $F_{1}$ plants from a cross between elf7-1 in Ws and elf7-2 in Col did not display the Colspecific phenotypes (data not shown). $\mathrm{F}_{2}$ plants displayed these phenotypes in a ratio of $3: 1$, respectively, suggesting that the additional phenotypes of elf7 mutations in $\mathrm{Col}$ are the result of a single recessive locus in the Col genetic background.

A phenotype of elf7 mutants in both the Col and Ws backgrounds is that petal number per flower often varied from three to five (the wild-type flower has four petals) (Fig. 1I,L). For example, 16\% of flowers had three petals and $17 \%$ had five petals in the elf7-1 mutant in Ws grown in LD, whereas in the same growth conditions $<6 \%$ of wild-type Ws flowers displayed altered petal number.

\section{ELF7 encodes the plant relative of yeast PAF1}

ELF7 is a single-copy gene in the Arabidopsis genome that consists of 12 exons encoding a predicted 589 -amino acid protein. The T-DNA in elf7-1 was inserted immediately upstream of the translation start codon (Fig. 2A); T-DNAs in elf7-2 and elf7-3 were inserted in the eighth and the last intron, respectively (Fig. 2A). The full-length ELF7 mRNA was not detectable in the elf7 mutants (Fig. 2C).

Except for the N-terminal region, the ELF7 protein is similar $(\sim 30 \%)$ over its entire length to PAF1, which was first described in Saccharomyces cerevisiae (Fig. 2B; the
Figure 2. ELF7 encodes the Arabidopsis homolog of yeast PAF1. (A) ELF7 gene structure. Exons are represented by filled boxes, and introns by lines. Triangles indicate T-DNA insertions. $(B)$ Amino acid sequence alignment of $A$. thaliana ELF7 (AtELF7) with $S$. cerevisiae PAF1 (ScPAF1), C. elegans PAF1 like (CePAL), and Oryza sativa PAF1 like (OsPAL). Numbers refer to amino acid residues; identical residues are shaded with black, while similar residues are shaded with gray. (C) ELF7 steady-state mRNA levels in seedlings determined by RNA blot analysis. About 15 $\mu \mathrm{g}$ of total RNA extracted from 10-d-old seedlings was loaded in each lane.
A

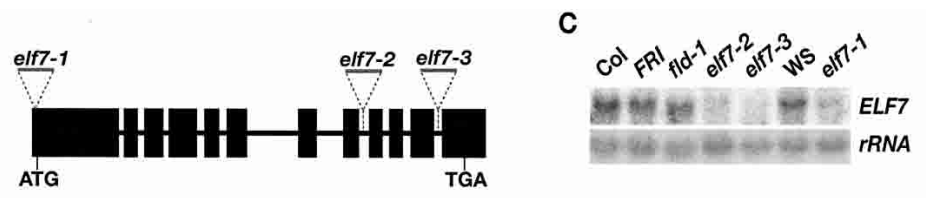

B

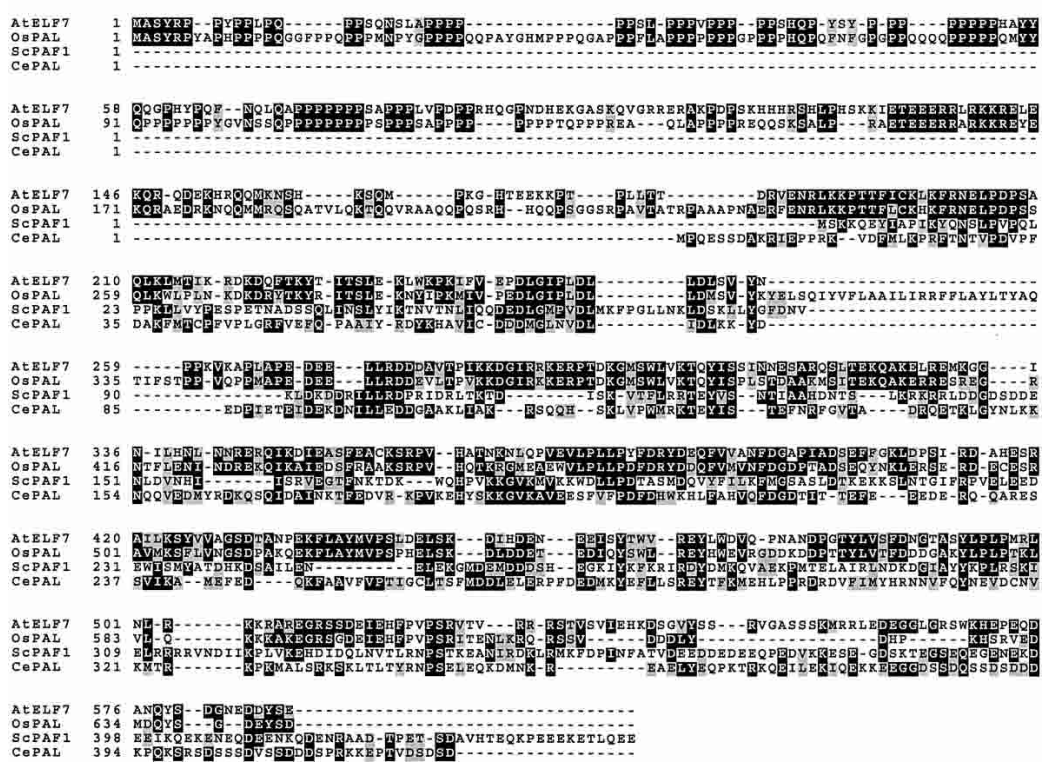


A

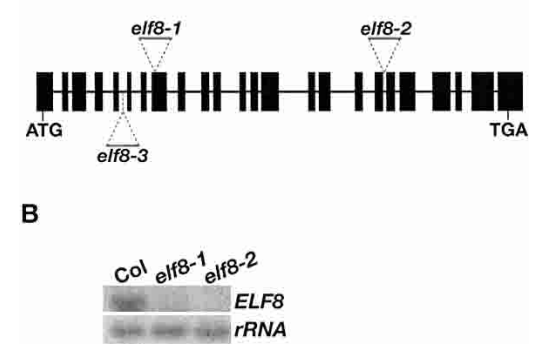

Figure 3. Structure and expression of ELF8. (A) ELF8 gene structure. (B) RNA blot analysis of ELF8 steady-state mRNA levels in seedlings.

Caenorhabditis elegans PAF1 is also included for comparison; Shi et al. 1996). The Arabidopsis PAF1 homolog has an $\mathrm{N}$-terminal extension that is highly proline-rich (46 prolines in the 94-amino acid region); this region is not found in PAF1s from yeast, C. elegans, Drosophila, or mammals. This $\mathrm{N}$-terminal extension is also present in the rice PAF1 homolog (Fig. 2B), indicating that this extension may be a unique feature of PAF1 proteins in higher plants.

Lesions in ELF8, a homolog of another PAF1 complex component, also cause early flowering

In S. cerevisiae (Sc), PAF1 is an integral component of a five-member complex consisting of PAF1, CTR9, LEO1, RTF1, and CDC73 called the PAF1 complex (Krogan et al. 2002; Squazzo et al. 2002). This complex associates with RNA polymerase II (Pol II) to regulate expression of a subset of the yeast genome (Betz et al. 2002; Ng et al. 2003). Searching the Arabidopsis protein sequence database revealed that Arabidopsis contains genes encoding proteins highly related to ScCTR9 and ScLEO1, but not to ScRTF1 and ScCDC73. At2g06210 (http://www. arabidopsis.org) is the Arabidopsis homolog of ScCTR9 (Supplementary Fig. S1).

We obtained three lines with T-DNA insertions in At2g06210 from the SALK T-DNA collection (Fig. 3A; Alonso et al. 2003). In both SD and LD, these three alleles caused early flowering similar to that of elf7 (Tables 2, 3). At2g06210 was therefore designated as ELF8. These elf 8 alleles also caused the leaf and flower phenotypes in the Col background described above for elf7 (Fig. 4; data not shown). $F_{1}$ complementation tests among the putative elf 8 alleles showed that all were allelic (data not shown), confirming the involvement of ELF8/At2g06210 in flowering-time regulation.
ELF8 is a single-copy gene in Arabidopsis that consists of 24 exons encoding a predicted 1064-amino acid protein. In elf8-1, the T-DNA was inserted into the eighth exon, and the T-DNAs in elf8-2 and elf8-3 were inserted into the eighteenth and the fifth intron, respectively (Fig. $3 \mathrm{~A})$. The full-length ELF8 mRNA was not detected in either elf8-1 or elf8-2 (Fig. 3B) (elf8-3 was not analyzed).

The Arabidopsis homolog of ScLEO1 is At5g61150. At5g61150 has been previously characterized as VIP4 (Zhang and van Nocker 2002). We isolated two vip4 alleles from the SALK T-DNA collection (Alonso et al. 2003; see Materials and Methods). When these two vip4 mutants are grown alongside e1f7, the phenotypes are identical (and similar to that previously described for other vip4 alleles) (Zhang and van Nocker 2002): the two SALK vip4 mutations cause early flowering in both LD and SD, and in the Col genetic background also cause the leaf and flower phenotypes that are observed in elf7 and elf8 (data not shown).

The similar phenotypes resulting from lesions in three genes that encode proteins that are homologous to constituents of the PAF1 complex in yeast suggests that the Arabidopsis proteins may be components of a similar complex in plants. Genetic evidence is consistent with this hypothesis: a double mutant between elf7 and elf8 flowers at the same time as the single mutants (Table 2). In addition, the double mutant was morphologically indistinguishable from either the elf7-3 or elf8-1 single mutants with respect to the leaf and flower development phenotypes (Fig. 4; data not shown).

\section{Lesions in elf7 and elf8 suppress FLC-dependent late flowering}

FLC, a MADS-box transcriptional factor, is a central regulator of the floral transition in Arabidopsis (Michaels and Amasino 1999; Sheldon et al. 1999). FLC is up-regulated by FRI and repressed by genes in the autonomous pathway (Michaels and Amasino 2001). Thus, either the presence of FRI or a lesion in an autonomous-pathway gene causes delayed flowering due to elevated FLC expression. To evaluate the genetic interaction of elf7 with $F R I$, elf7-2 was introduced into Col-FRI $I^{\text {sf }}$ (Lee et al. 1994), and to evaluate the interaction with an autonomous-pathway gene, elf7-2 was introduced into a line with a mutation in FLD (fld-1) (He et al. 2003). The lateflowering phenotypes of Col-FRI $I^{\text {f2 }}$ and fld-1 are completely suppressed by elf7-2 (Table 3 ). We also determined FLC transcript levels in Col, FRI, fld-1, elf7, and double mutants. FLC was expressed at a low level in Col, and, as previously shown, up-regulated in FRI and fld-1 (Fig. 5A,B). However, in elf7-2, elf7-3, elf7-2 FRI, and

Table 2. Primary leaf number at bolting of elf7, elf8, and elf7 elf8 mutants in short days

\begin{tabular}{lccccc}
\hline Col & elf8-1 & elf8-2 & elf8-3 & elf7-3 & elf8-1 elf7-3 \\
\hline $66.9 \pm 3.9$ & $15.2 \pm 1.6$ & $16.3 \pm 2.1$ & $17.1 \pm 1.7$ & $21.2 \pm 2.9$ & $15.0 \pm 0.9$ \\
$(10.7 \pm 1.5)$ & $(4.3 \pm 0.7)$ & $(4.0 \pm 0.7)$ & $(4.1 \pm 0.7)$ & $(5.6 \pm 0.8)$ & $(4.3 \pm 0.8)$ \\
\hline
\end{tabular}

Values shown are mean number \pm standard deviation of rosette and cauline leaves (in parentheses); 10 plants were scored for each line. 
He et al.

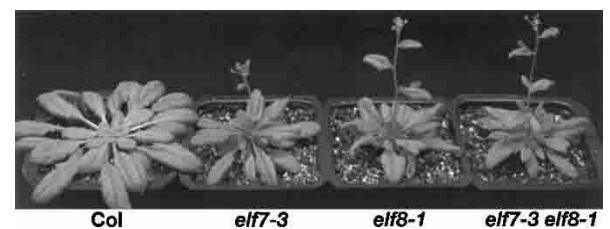

Figure 4. The phenotypes of elf7, elf 8 , and elf 7 elf 8 mutants in short days.

elf7-2 fld-1, FLC transcripts were not detectable (Fig. $5 \mathrm{~A}, \mathrm{~B})$. Thus, ELF7 activity is required for FLC expression. We also examined whether ELF7 expression was influenced by $F R I$ or the autonomous pathway. As shown in Figure 2C, ELF7 transcript levels were not regulated by FRI or FLD.

To evaluate whether the activity of ELF8 is also required for FLC expression, we introduced elf8-1 into Col$F R I^{s f 2}$ and another autonomous mutant, $f_{V e}-4$ (Michaels and Amasino 2001; Ausin et al. 2004). The late-flowering phenotypes of FRI and $f_{V e}-4$ were completely suppressed by elf8-1 (Table 3). RNA blot analysis showed that FLC transcripts were not detectable in elf8-1, elf8-2, elf8-1 FRI, and elf8-1 fve-4 (Fig. 5C). Thus, ELF8, like ELF7, is required for $F L C$ expression.

Lesions in elf7 and elf8 also affect flowering via other MADS-box genes

The elf7 and elf8 mutants flower earlier in both LD and SD than plants containing an flc-null mutant, flc-3 (Fig. 6A). Thus, ELF7 and ELF8 must participate in at least one $F L C$-independent flowering pathway in addition to their role in FLC expression. The early flowering of elf7 and elf8 mutants in SD is reminiscent of the phenotype caused by lesions in another MADS-box gene, FLM (Ratcliffe et al. 2001; Scortecci et al. 2001). FLM is closely related to $F L C$ and, like $F L C, F L M$ also acts as a repressor of flowering. However, unlike FLC, FLM is involved in the photoperiod regulation of flowering (Scortecci et al. 2003). We examined FLM expression in both elf7-2 and elf8-1 plants, and found that FLM expression was suppressed in both mutants compared to wild type (Fig. 6B). Therefore, ELF7 and ELF8 are required for expression of both FLC and FLM. A MADS-box gene that is likely to encode a partner of FLM is SUPPRESSOR OF VEGETATIVE PHASE (SVP) (Scortecci et al. 2003). SVP is in a different clade of MADS-box genes from FLC and FLM. Lesions in elf7 or elf8 had no effect on SVP expression (Fig. 6B). We also examined expression of several MADSbox genes that do not affect flowering as additional controls. As in the situation for $S V P$, the mRNA levels of AGAMOUS LIKE 16 (AGL16), AGL18, and AGL30 are not affected by lesions in elf7 or elf8 (Fig. 6C). Thus, ELF7 and ELF8 may regulate select clades of MADS-box genes. Another MADS-box gene in the FLC/FLM clade is $M A F 2$, a repressor of floral transition (Ratcliffe et al. 2003), and consistent with the common regulation of this clade, MAF2 expression is also suppressed in elf7 and elf8 mutants (Fig. 6B).
Table 3. Primary leaf number at bolting of elf7, elf8, and double mutants in long days

\begin{tabular}{lrc}
\hline Lines & $\begin{array}{c}\text { Rosette } \\
\text { leaf number }\end{array}$ & $\begin{array}{c}\text { Cauline } \\
\text { leaf number }\end{array}$ \\
\hline Col & $12.2 \pm 1.2$ & $3.2 \pm 0.7$ \\
flc-3 & $10.3 \pm 1.6$ & $2.4 \pm 0.5$ \\
elf7-2 & $6.6 \pm 0.7$ & $2.8 \pm 0.7$ \\
elf8-1 & $6.5 \pm 0.5$ & $3.5 \pm 0.5$ \\
FRI & $67.0 \pm 7.8$ & $9.8 \pm 2.1$ \\
elf7-2 FRI & $6.7 \pm 0.9$ & $3.3 \pm 0.7$ \\
elf8-1 FRI & $6.9 \pm 0.7$ & $3.3 \pm 0.5$ \\
fld-1 & $52.6 \pm 11$ & $9.0 \pm 1.9$ \\
fld-1 elf7-2 & $7.0 \pm 0.7$ & $3.1 \pm 0.8$ \\
fve-4 & $44.0 \pm 4.1$ & $8.5 \pm 1.3$ \\
fve-4 elf8-1 & $7.4 \pm 0.9$ & $3.6 \pm 0.7$ \\
\hline
\end{tabular}

Values shown are mean number \pm standard deviation; 10 plants were scored for each line.

\section{ELF7 and ELF8 are expressed in the shoot apex}

Given that ELF7 and ELF8 are both required for FLC and FLM expression, it was of interest to compare the spatial expression patterns of these genes to that of FLC and FLM. Accordingly, fusions to the reporter gene $\beta$-glucuronidase (GUS) were made for each gene (see Materials and Methods). ELF7 expression is highest in regions that contain dividing cells in the shoot and root apex; this pattern is the same as that of FLC (Fig. 7A, panels $\mathrm{a}, \mathrm{b}$; also Michaels and Amasino 2000) and FLM (Scortecci et al. 2001). The ELF8 construct produced an expression pattern that overlapped with that of ELF7 and $F L C$, but it was also readily detected in all root tissues and in regions around hydathodes at the tips of leaves (Fig. 7A, panel c). We examined the steady-state mRNA levels of ELF7, ELF8, and FLC in seedlings by RT-PCR.

A

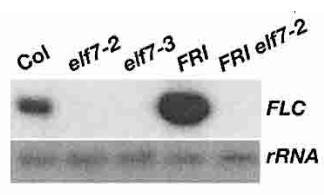

B

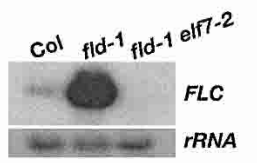

C

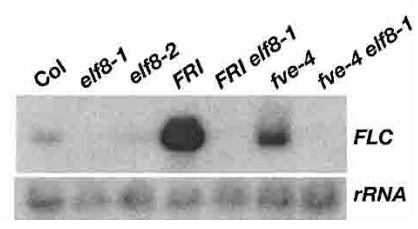

Figure 5. elf7 and elf8 suppress FLC expression. (A) Steadystate FLC mRNA levels in elf7 fri and elf7 FRI seedlings $(10 \mathrm{~d}$ old) as determined by RNA blot. (B) RNA blot analysis of FLC mRNA levels in elf7 fld-1 seedlings. (C) Steady-state $F L C$ mRNA levels in elf8, elf8 FRI, and elf $f_{V} e-4$ seedlings as determined by RNA blot. 
A
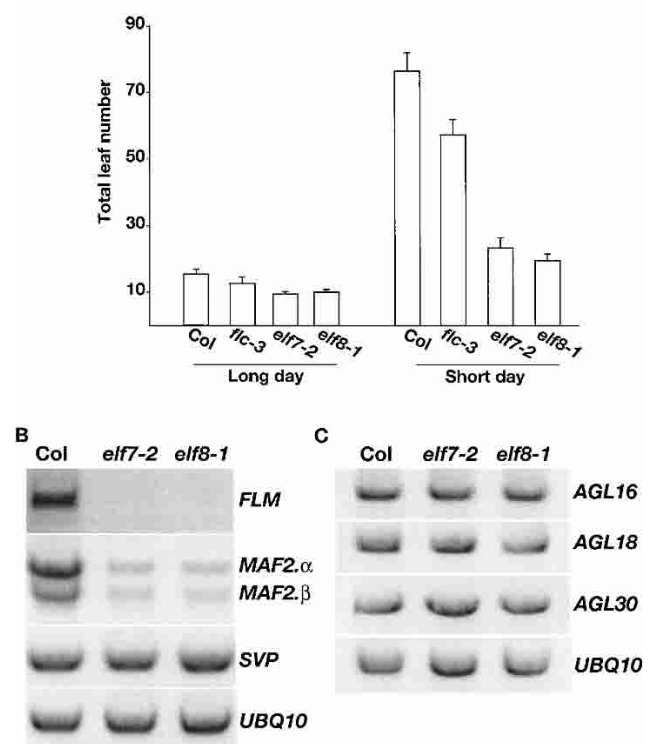

Figure 6. $F L C$-independent early flowering in elf7 and elf8. $(A)$ Flowering times of $f l c$, elf7, and elf8 in long days and short days. Bars represent mean values \pm standard deviation of total leaf number at bolting. For each line, 10 plants were scored. $(B)$ RT-PCR analysis of expression of FLC relatives in elf7 and elf8. Two variants of MAF2 were detected. UBQ10 was used as a control. (C) RT-PCR analysis of expression of additional MADS-box genes in elf7 and elf8.

Consistent with the reporter gene patterns, all three genes are expressed in the shoot apex, where the floral transition occurs (Fig. 7B).

Histone 3 is hypermethylated at Lys 4 in actively transcribed FLC chromatin

The yeast PAF1 complex associates with RNA Pol II and is thought to regulate gene expression by recruiting SET1 (a histone 3 Lys 4 [H3-K4] methyl transferase) to the initially transcribed $\left(5^{\prime}\right)$ regions of target-gene chromatin (Krogan et al. 2003; Ng et al. 2003). This generates H3-K4 trimethylation predominantly in the $5^{\prime}$ portion of transcribed regions ( $\mathrm{Ng}$ et al. 2003), and this chromatin modification is a hallmark of actively transcribed genes in S. cerevisiae (Santos-Rosa et al. 2002).

Because ELF7 and ELF8 are relatives of yeast PAF1 and CTR9, respectively, we evaluated whether a mechanism similar to that mediated by the yeast PAF1 complex might be involved in FLC expression. The H3-K4 trimethylation state of FLC chromatin in an FRI-containing line (in which FLC is actively expressed) was compared to that in Col (which lacks FRI and does not express FLC at high levels) by chromatin immunoprecipitation (ChIP) assays using an antibody directed against trimethylated $\mathrm{K} 4$ of histone $\mathrm{H} 3$. Similar to the situation observed for PAF1-regulated genes in yeast, a region of FLC chromatin around the start site of transcription is enriched in $\mathrm{H} 3-\mathrm{K} 4$ trimethylation when FLC is up-regulated by FRI (Fig. 8). The FRI-mediated increase in H3-K4 trimethylation of FLC chromatin did not occur in elf7 or elf8 mutants (Fig. 9A,B). Therefore, the FRI-enhanced H3-K4 trimethylation of FLC chromatin is dependent on these two relatives of PAF1 complex components. The H3-K4 trimethylation level of FLC chromatin is also increased in the autonomous-pathway mutant fld (Fig. 9A,B).

$\mathrm{H} 3-\mathrm{K} 4$ trimethylation in the $5^{\prime}$ portion of the transcribed region of FLM chromatin was also greatly reduced in elf7-2 and elf8-1 mutants compared to wildtype Col (Fig. 9C,D), indicating that ELF7 and ELF8 are involved in H3-K4 trimethylation of two members of this clade of MADS-box genes.

A conserved region located in the $5^{\prime}$-UTR of MADS-box genes is required for FLC expression

Because the enhanced trimethylation occurred in the $5^{\prime}$ UTR of $F L C$, it was of interest to determine whether this region plays a role in FLC transcription. We first compared the sequence of this region of $F L C$ with that of other members of the FLC clade: FLM, MAF2, and Brassica napus FLC1 (an Arabidopsis FLC ortholog) (Tadege et al. 2001), and found a conserved 34-bp motif at the same 5' location in all of these genes (Fig. 10A). This motif was not found in $S V P$, a gene that is likely to be a partner of FLM but that is in a different clade of MADSbox genes (Scortecci et al. 2003), nor was it found in other MADS-box genes that affect flowering. We created an internal 80-bp deletion $(\Delta 80)$ that spanned this 34-bp motif within the $5^{\prime}$-UTR of $F L C$ (Fig. 10B; -4 to -83 , A of the start codon ATG is +1 ). This $\Delta 80$ construct preserves a 26-bp region immediately downstream of the transcription start point and a 7-bp region immediately upstream of the translation start codon. The $\Delta 80$ construct and the

A

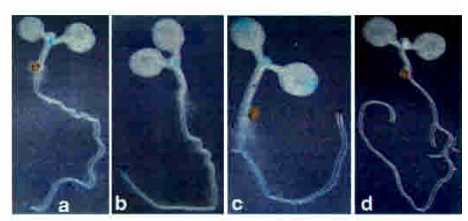

B

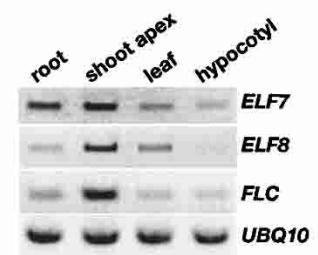

Figure 7. Spatial expression pattern of FLC, ELF7, and ELF8 in seedlings. (A) Spatial expression pattern of GUS reporter gene fused to FLC, ELF7, or ELF8 in seedlings. (Panel a) FLC:GUS in Col $F R I^{s f 2}$. (Panel b) ELF7:GUS in Col. (Panel c) ELF8:GUS in Col. (Panel d) FLC:GUS in elf7-2 FRI. (B) RT-PCR analysis of expression of $F L C, E L F 7$, and ELF8 in various tissues from seedlings. 
He et al.

A

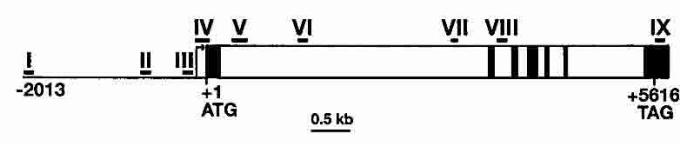

B

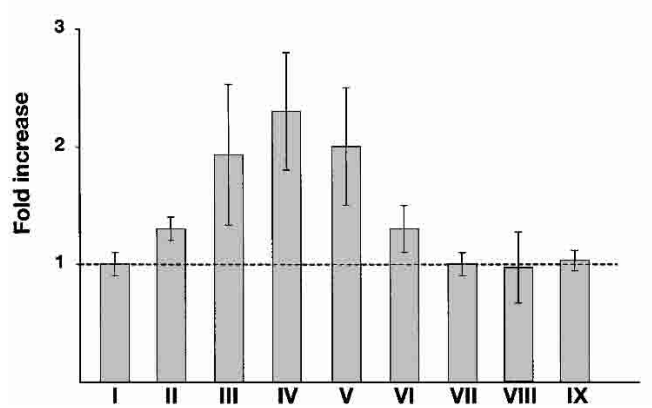

Figure 8. ChIP analysis of the H3-K4 trimethylation level of FLC chromatin. (A) Schematic structure of FLC. I to IX represent the regions in which $\mathrm{K} 4$ trimethylation states were examined by ChIP. The translation initiation point is +1 . The filled boxes represent exons, and open boxes represent introns. The arrow indicates transcription start point. $(B)$ Relative levels of trimethylated $\mathrm{K} 4$ at various genomic FLC regions in Col FRI $I^{\text {ff2 }}$. Three independent immunoprecipitations were performed; the immunoprecipitated DNA was quantified using PCR. The fold enrichments of $\mathrm{Col} F R I^{s f 2}$ over $\mathrm{Col}$ at indicated regions are shown. The values shown are mean \pm standard deviation.

wild-type $F L C$ construct $(-1167)$ were introduced into flc-3, flc-3 FRI, and flc-3 fpa (FPA is a repressor of FLC expression in the autonomous pathway). The wild-type $(-1167)$ transgene behaved as expected: in FRI and fpa backgrounds, $T_{1}$ transformants harboring this transgene were late flowering (Fig. 10C) and had high levels of FLC expression (Fig. 10D). However, the $\Delta 80$ transgene did not repress flowering, nor was it up-regulated in FRI or fpa (Fig. 10C,D). Therefore, the region containing the conserved motif within the $5^{\prime}$-UTR of $F L C$ is required for FLC expression, although these data do not address whether this region is directly required for ELF7 and ELF8 to promote $F L C$ expression.

\section{Discussion}

In this study, we identified mutations in two genes, elf7 and $e l f 8$, that cause rapid flowering in several situations in which flowering is otherwise delayed. One situation is that elf7 and elf8 suppress the delayed flowering of both $F R I$-containing winter annuals and autonomous-pathway mutants. The presence of $F R I$ or autonomous-pathway mutations delays flowering by increasing $F L C$ expression, and we have shown that ELF7 and ELF8 are required for this increase. Thus, ELF7 and ELF8 are required to elevate $F L C$ expression to a level that creates the vernalization-responsive, winter-annual habit (Michaels and Amasino 2000).

The elf7 and elf8 lesions also suppress the delayed flowering that results from growth under noninductive photoperiods. Because loss of FLC activity has only a minor effect on flowering in noninductive photoperiods (Michaels and Amasino 2001), other genes must be involved in this aspect of the elf7 and elf8 flowering phenotype. elf7 and elf8 flower early in noninductive photoperiods, at least in part because FLM and MAF2 expression also requires ELF7 and ELF8. FLM is the closest relative of FLC in Arabidopsis (Ratcliffe et al. 2001; Scortecci et al. 2001); both are flowering repressors, but, unlike FLC, FLM acts in the photoperiod pathway (Scortecci et al. 2003). MAF2 is another MADS-box gene in which mutations affect photoperiod-dependent flowering (Ratcliffe et al. 2003). Thus, we have a molecular explanation for the broad effects of elf7 and elf8 lesions on flowering behavior: ELF7 and ELF8 are required for the expression of members of a clade of MADS-box genes that are involved in several flowering pathways.

ELF7 encodes the Arabidopsis homolog of yeast PAF1, and ELF8 encodes the relative of yeast CTR9. In yeast,

A

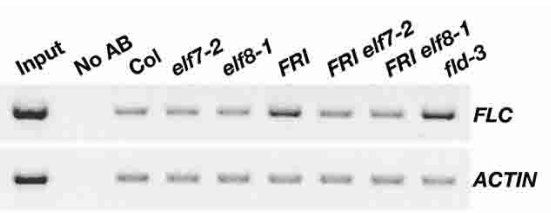

B

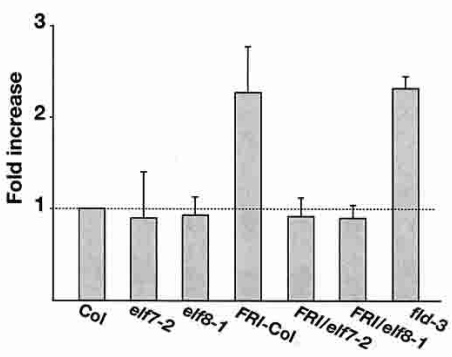

C

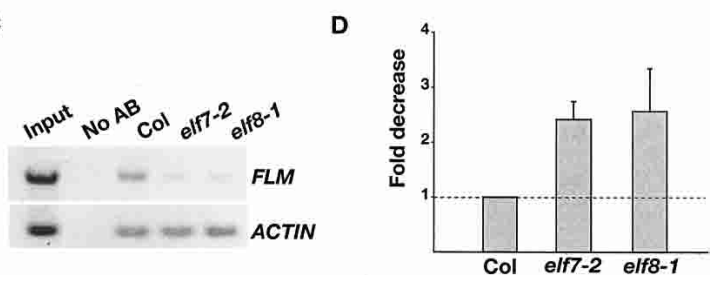

Figure 9. ELF7 and ELF8 are required for H3-K4 hypertrimethylation in FLC and FLM chromatin. (A) ChIP analysis of H3-K4 trimethylation state of Region IV (see Fig. 8A) in FLC chromatin of elf7, elf8, and related double mutants. A representative ChIPPCR is shown. The input is Col chromatin before immunoprecipitation. "No $\mathrm{AB}^{\prime}$ refers to the control sample lacking antibody. ACTIN served as an internal control. (B) Relative levels of trimethylated K4 at Region IV in FLC chromatin of elf7, elf8, and related double mutants. Three independent immunoprecipitations were performed; the immunoprecipitated DNA was quantified using PCR. The level of trimethylated K4 in each mutant or FRI-containing line was normalized to that in Col (the level of trimethylated K4 in Col was arbitrarily set at 1.0). The values shown are mean \pm standard deviation. (C) ChIP analysis of H3-K4 trimethylation state of the 5'-transcribed region of FLM chromatin in elf7 and elf8. (D) Relative levels of trimethylated $\mathrm{K} 4$ at the $5^{\prime}$ region of FLM chromatin in elf7 and elf8. The relative levels were calculated as described in $B$. 
A

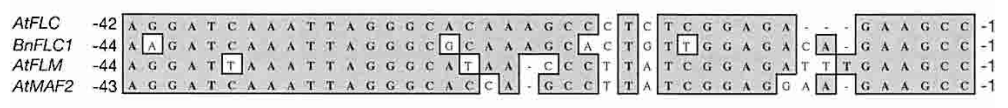

в

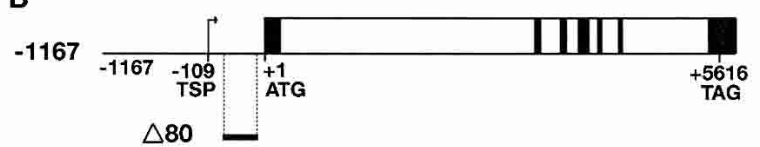

c

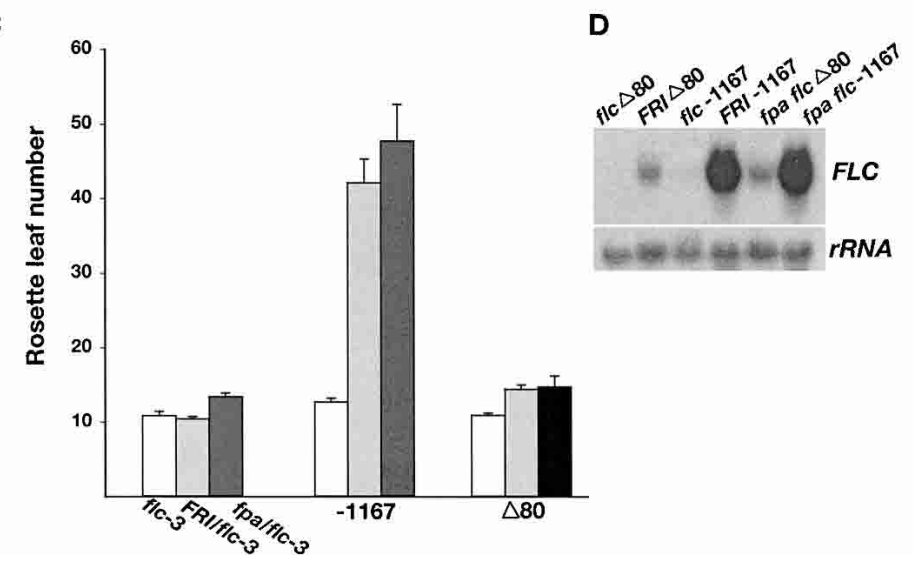

Figure 10. An 80 -bp deletion $(\Delta 80)$ in the $5^{\prime}$-untranslated region (UTR) of FLC suppresses its expression. (A) 5'-UTR sequence alignment of A. thaliana FLC $(A t F L C)$ with $B$. napus FLC 1 (BnFLC1), AtFLM, and AtMAF2. The translation initiation point is +1 . $(B)$ Schematic structure of a functional FLC transgene $(-1167)$ and the $\Delta 80$ transgene. The translation initiation point is +1 . The filled boxes represent exons, and open boxes represent introns. The arrow indicates transcription start point. (C) Flowering times of $T_{1}$ transgenic plants derived from transgenes -1167 and $\Delta 80$. For each transgenic line, 15-33 $T_{1}$ plants were scored. The values shown are mean \pm standard error. White, gray, and black boxes represent fri flc-3, FRI flc-3, and fpa flc-3 genetic backgrounds, respectively. (D) Abundance of steady-state $F L C$ mRNA levels in transgenic seedlings determined by RNA blot.
PAF1 and CTR9 are members of a complex of five proteins, called the PAF1 complex, that associates with RNA Pol II (Squazzo et al. 2002; Krogan et al. 2003; Ng et al. 2003). The other members of this complex are LEO1, RTF1, and CDC73 (Squazzo et al. 2002). To date, a homolog of only one other complex member, LEO1, has been identified in Arabidopsis (Zhang and van Nocker 2002). Lesions in this homolog, VIP4, create a phenotype similar to that of elf7 and elf8. The similar phenotype of single mutants in three single-copy plant homologs of components of the yeast PAF1 complex is consistent with ELF7, ELF8, and VIP4 operating in a similar complex in plants. The observation that a double mutant between elf7 and elf8 is identical to the elf8 single mutant provides further support for this model (as does the effect on target-gene chromatin discussed below). The closest Arabidopsis relatives of RTF1 and CDC73 are Atlg61040 and At3g22590, respectively. We have examined a T-DNA insertion mutant in At3g22590, and the insertion in this gene does not cause any effect on flowering, nor does it cause any of the other phenotypes of elf7, elf8, or vip4 mutants. One possibility is that At3g22590 is not a part of the PAF1 complex in Arabidopsis. Another possibility is that this protein is a part of the complex, but its loss of function in Arabidopsis does not impair the ability of the complex to regulate flowering. In this regard, it is interesting to note that the plant PAF1 proteins are predicted to encode a proline-rich $\mathrm{N}$ terminal region that is not present in PAF1 proteins from animals and yeast. Perhaps this N-terminal region may permit interaction with other components of the complex that are unique to plants.

Recently it has been demonstrated that the yeast PAF1 complex is involved in recruiting SET1 to the RNA Pol II elongation complex (Krogan et al. 2003; Ng et al. 2003). SET1 is a methyltransferase that catalyzes methylation of histone $3(\mathrm{H} 3)$ on Lys $4(\mathrm{~K} 4)$ to produce trimethylated H3-K4 (Roguev et al. 2001; Santos-Rosa et al. 2002). Typically the regions of chromatin in a transcription unit that are transcribed early become enriched in H3-K4 trimethylation, and this chromatin modification is thought to provide a positive epigenetic mark to maintain a gene in an actively transcribed state (Santos-Rosa et al. 2002; Ng et al. 2003). We have shown that ELF7 and ELF8 are required for H3-K4 trimethylation of the chromatin of FLC and FLM, and that elf7 and elf8 lesions attenuate FLC and FLM expression. Furthermore, we have identified an Arabidopsis relative of the Drosophila ASH1 (Beisel et al. 2002; Byrd and Shearn 2003) and yeast SET1 H3-K4 methyltransferases (Roguev et al. 2001; Santos-Rosa et al. 2002). Mutations in this gene, EARLY FLOWERING IN SHORT DAYS (EFS) (Soppe et al. 1999), also reduce $\mathrm{H} 3-\mathrm{K} 4$ trimethylation in $F L C$ chromatin and cause an early-flowering phenotype similar to that in elf7, elf8, and vip4 (S.D. Michaels, Y. He, and R.M. Amasino, unpubl.). Thus, members of both the yeast and the plant PAF1 complexes appear to regulate target genes by a similar mechanism.

In the Ws genetic background, elf7 mutants developed normally except for early flowering and altered petal number, which suggests that only a small number of genes were strongly affected by loss of ELF7 activity. In Col, elf7 mutants exhibit other developmental abnormalities including smaller leaves and sepals, although the Col mutants complete the normal plant life cycle. The lack of lethality in Arabidopsis plants that have lost 
ELF7 or ELF8 activity, despite the fact that ELF7 and ELF8 are encoded by single-copy genes, is similar to the phenotype of lesions in the corresponding genes in yeast. Disruption of the PAF1 complex in yeast is not lethal, and only the abundance of a small subset of mRNAs is affected (Betz et al. 2002).

Little is known about why the expression of certain genes is affected more strongly than others by loss of PAF1 complex activity. In the examples we have studied, the clade of MADS-box genes appears to be the key feature for ELF7 and ELF8 involvement rather than the flowering pathway in which the MADS-box gene participates. Specifically, FLM and SVP are in the same flowering pathway (Scortecci et al. 2003) but are in different clades of MADS-box genes, and FLM but not SVP expression is affected by lesions in elf7 and elf8. However, expression of FLC, FLM, and MAF2, which are in the same clade but affect different flowering pathways, is attenuated by lesions in elf7 and elf8. Members of the FLC clade, including a Brassica napus gene, have a conserved nucleotide sequence in the $5^{\prime}$-UTR that is within the region of the highest level of $\mathrm{H} 3-\mathrm{K} 4$ trimethylation. This conserved region is not present in SVP or in MADS-box genes in other clades that are involved in flowering time regulation. A deletion of this conserved region prevents FLC up-regulation by FRI or autonomous-pathway mutations. These data are consistent with this conserved region playing a role in the ELF7/ELF8-mediated activation of the FLC clade of MADS-box genes, but a direct demonstration of this will require further experiments.

Covalent modifications of chromatin proteins constitute a "histone code" for maintaining states of gene activation or repression (Iizuka and Smith 2003). These modifications recruit transcriptional activators and repressors involved in the regulation of gene expression and the maintenance of certain histone modifications. Trimethylation of $\mathrm{H} 3-\mathrm{K} 4$ in the 5 ' regions of genes is thought to promote gene expression in yeast by recruiting ISW1p (Santos-Rosa et al. 2003), a yeast ATP-hydrolyzing, chromatin-remodeling protein that may be involved in nucleosome positioning (Tsukiyama et al. 1999). An Arabidopsis relative of ISW1p, PIE1, is a candidate for the plant ATP-dependent chromatin-remodeling protein that is required for expression of genes regulated by ELF7 and ELF8: mutations in PIE1 suppress FLC expression and cause early flowering in both LD and SD and altered petal number, and pie alleles in the Col genetic background display additional phenotypes similar to those of elf7 and elf8 such as small leaves (Noh and Amasino 2003). The phenotypic similarity among pie1, elf7, elf8, and vip4 mutants indicates that all of these genes are in the same pathway and regulate similar target genes. We propose that PIE1 binds the $5^{\prime}$ region of FLC, FLM, and MAF2 chromatin when these chromatin regions are enriched for $\mathrm{H} 3-\mathrm{K} 4$ trimethylation resulting from ELF7/ELF8-dependent recruiting of the EFS methyltransferase.

This study provides the first molecular correlation of a specific FLC chromatin modification and the increased $F L C$ expression that occurs in lines that behave as winter annuals because of the presence of FRI or autonomouspathway mutations. In Col, which has a nonfunctional fri allele (Johanson et al. 2000), FLC is not highly expressed, nor is FLC chromatin highly trimethylated at H3-K4. The loss of an autonomous-pathway negative regulator of FLC such as FLD permits FLC up-regulation and increased $\mathrm{H} 3-\mathrm{K} 4$ trimethylation in an fri background. Thus autonomous-pathway components act, at least in part, to restrict the ability of ELF7 and ELF8 to activate $F L C$ and increase $\mathrm{H} 3-\mathrm{K} 4$ trimethylation. In the presence of a dominant allele of FRI, the ability of the autonomous pathway to prevent FLC up-regulation and increased H3-K4 trimethylation is blocked. Additional studies are required to determine whether the antagonistic activities of FRI and the autonomous pathway converge on the ELF7/ELF8-mediated H3-K4 methylation of FLC chromatin, or whether this FLC chromatin modification is downstream of FRI and the autonomous pathway.

\section{Materials and methods}

\section{Plant materials and growth conditions}

flc-3 (Michaels and Amasino 1999), Col FRI $I^{\text {sf2 }}$ (the functional FRI from Arabidopsis accession San Feliu-2 introgressed into Col) (Lee et al. 1994), fve-4 (Ausin et al. 2004), fld-1 (Sanda and Amasino 1996), fld-3 (He et al. 2003), FRI flc-3 (Michaels and Amasino 2001), and fpa flc-3 (Michaels and Amasino 2001) were described previously. The vip4 alleles were isolated from the SALK T-DNA collection (SALK_122755 and SALK_039374) (Alonso et al. 2003). Plants were grown either under white fluorescent light in short days ( $8 \mathrm{~h}$ light $/ 16 \mathrm{~h}$ night) or in long days (16 h light $/ 8 \mathrm{~h} \mathrm{night)} \mathrm{at} 22^{\circ} \mathrm{C}$.

\section{Plasmid construction}

To construct ELF7:GUS, a 3.2-kb ELF7 genomic fragment including a $1.0-\mathrm{kb}$ region $5^{\prime}$ of the transcription start point and 2.2 $\mathrm{kb}$ of coding sequence was fused to the GUS coding sequence. To construct ELF8:GUS, a $2.0-\mathrm{kb}$ region $5^{\prime}$ of the transcription start point was fused to the GUS coding region. The 80-bp internal deletion of the $5^{\prime}$-UTR of FLC was made by a PCR-mediated fusion strategy.

RNA blot analyses

Total RNA was isolated from 10-d-old seedlings using TRI Reagent as recommended by the manufacturer (Sigma). About 15 $\mu \mathrm{g}$ of total RNA was loaded in each lane, separated in a denaturing formaldehyde agarose gel, and transferred to a Hybond $\mathrm{N}^{+}$ membrane (Amersham Pharmacia). The FLC 3'-UTR fragment was used as a probe for FLC detection. Blots were subsequently probed with an $18 \mathrm{~S}$ rDNA probe for comparison of the quantity of RNA loaded among samples.

\section{$R T-P C R$}

cDNAs were synthesized from $2.0 \mu \mathrm{g}$ of total RNA with Superscript Reverse Transcriptase (Life Technologies). The FLM, SVP, and $U B Q 10$ cDNAs were amplified as described previously (Scortecci et al. 2003). The cDNAs of AGL16, AGL18, and AGL30 were amplified as described previously by Parenicova et al. (2003). The $M A F 2$ cDNA was amplified with the primer pair 
MAF2f (5'-GGGTAGAAAAAAAGTCGAGATCAAGCGA-3') and MAF2r (5'-CTTGAGCAGCGGAAGAGTCTCC-3'). The primer pair ELF7f (5'-TCTCAGATGCCCAAGGGACAC-3') and ELF7r $\left(5^{\prime}\right.$-GGATGCTTCAATATCCTTGATTTGT-3') was used to amplify ELF7 cDNA; the primer pair ELF8f (5'-CGGGT ACAAGGACACGGGCAT-3') and ELF8r (5'-CAAGGCTCA ATCCAAATAACCCAAAATACA-3') was used to amplify ELF8 cDNA; the primer pair FLCf $\left(5^{\prime}\right.$-TTCTCCAAACGTCG CAACGGTCTC- $\left.3^{\prime}\right)$ and FLCr (5'-GAGCTTTTGACTGATGA TCCAAGGCTTTA-3') was used to amplify FLC cDNA.

\section{$\beta$-Glucuronidase staining in plants}

Histochemical $\beta$-glucuronidase staining was performed as described previously (Schomburg et al. 2001). FLC:GUS-, ELF7:GUSand ELF8:GUS-containing seedlings were stained for $3 \mathrm{~h}$ at $37^{\circ} \mathrm{C}$.

\section{ChIP assays}

The chromatin immunoprecipitation experiments were performed as described by Johnson et al. (2002) using 10-d-old seedlings. The anti-trimethylated $\mathrm{K} 4$ histone $\mathrm{H} 3$ antibodies and the ChIP assay kit were from Upstate Biotechnology. Each of the immunoprecipitations was replicated three times. The amounts of genomic DNA immunoprecipitated were determined by quantitative PCR. The primer pair $\mathrm{CH} 2\left(5^{\prime}\right.$-GTTCTCAATTC GCTTGATTTCTAGT-3') and CH12 (5'-GGCCCGACGA AGAAAAAGTAGATAGGC-3') was used to amplify Region IV of $F L C$; the primer pair FLMf (5'-CCGGAGATTAGGATTA AATTAGGGCATA-3') and FLMr (5'-CTAAGCAATACAAA GATTACTTAAACAACATC-3') was used to amplify FLM; the primer pair used to amplify ACTIN 2/7 was described previously (Johnson et al. 2002). Aliquots of the PCR reactions were resolved by electrophoresis in $1.5 \%$ agarose gels, and quantified with ImageQuant software (Molecular Dynamics, Inc).

\section{Acknowledgments}

We thank the ABRC at Ohio State for providing T-DNA-insertion lines in the Col background, and Scott T. Woody, Robert J. Schmitz, and Sibum Sung for critical reading this manuscript. This work was supported by the College of Agricultural and Life Sciences and the Graduate School of the University of Wisconsin, and by National Science Foundation grants 0133663 and 0209786 to R.M.A.

\section{References}

Alonso, J.M., Stepanova, A.N., Leisse, T.J., Kim, C.J., Chen, H., Shinn, P., Stevenson, D.K., Zimmerman, J., Barajas, P., Cheuk, R., et al. 2003. Genome-wide insertional mutagenesis of Arabidopsis thaliana. Science 301: 653-657.

Ausin, I., Alonso-Blanco, C., Jarillo, J.A., Ruiz-Garcia, L., and Martinez-Zapater, J.M. 2004. Regulation of flowering time by FVE, a retinoblastoma-associated protein. Nat. Genet. 36: $162-166$.

Bastow, R., Mylne, J.S., Lister, C., Lippman, Z., Martienssen, R.A., and Dean, C. 2004. Vernalization requires epigenetic silencing of FLC by histone methylation. Nature 427: 164167.

Beisel, C., Imhof, A., Greene, J., Kremmer, E., and Sauer, F. 2002. Histone methylation by the Drosophila epigenetic transcriptional regulator Ash1. Nature 419: 857-862.

Betz, J.L., Chang, M., Washburn, T.M., Porter, S.E., Mueller,
C.L., and Jaehning, J.A. 2002. Phenotypic analysis of Paf1/ RNA polymerase II complex mutations reveals connections to cell cycle regulation, protein synthesis, and lipid and nucleic acid metabolism. Mol. Genet. Genomics 268: 272285.

Blazquez, M.A., Ahn, J.H., and Weigel, D. 2003. A thermosensory pathway controlling flowering time in Arabidopsis thaliana. Nat. Genet. 33: 168-171.

Boss, P.K., Bastow, R.M., Mylne, J.S., and Dean, C. 2004. Multiple pathways in the decision to flower: Enabling, promoting, and resetting. Plant Cell 16 Suppl: S18-S31.

Burn, J.E., Smyth, D.R., Peacock, W.J., and Dennis, E.S. 1993. Genes conferring late flowering in Arabidopsis thaliana. Genetica 90: 147-155.

Byrd, K.N. and Shearn, A. 2003. ASH1, a Drosophila trithorax group protein, is required for methylation of lysine 4 residues on histone H3. Proc. Natl. Acad. Sci. 100: 1153511540.

Clarke, J.H. and Dean, C. 1994. Mapping FRI, a locus controlling flowering time and vernalization response in Arabidopsis thaliana. Mol. Gen. Genet. 242: 81-89.

Gazzani, S., Gendall, A.R., Lister, C., and Dean, C. 2003. Analysis of the molecular basis of flowering time variation in Arabidopsis accessions. Plant Physiol. 132: 1107-1114.

He, Y., Michaels, S.D., and Amasino, R.M. 2003. Regulation of flowering time by histone acetylation in Arabidopsis. Science 302: 1751-1754.

Hepworth, S.R., Valverde, F., Ravenscroft, D., Mouradov, A., and Coupland, G. 2002. Antagonistic regulation of flowering-time gene SOC1 by CONSTANS and FLC via separate promoter motifs. EMBO J. 21: 4327-4337.

Iizuka, M. and Smith, M.M. 2003. Functional consequences of histone modifications. Curr. Opin. Genet. Dev. 13: 154-160.

Johanson, U., West, J., Lister, C., Michaels, S., Amasino, R., and Dean, C. 2000. Molecular analysis of FRIGIDA, a major determinant of natural variation in Arabidopsis flowering time. Science 290: 344-347.

Johnson, L., Cao, X., and Jacobsen, S. 2002. Interplay between two epigenetic marks. DNA methylation and histone $\mathrm{H} 3$ lysine 9 methylation. Curr. Biol. 12: 1360-1367.

Koornneef, M., Blankestijn-de Vries, H., Hanhart, C., Soppe, W., and Peeters, T. 1994. The phenotype of some late-flowering mutants is enhanced by a locus on chromosome 5 that is not effective in the Landsberg erecta wild-type. Plant J. 6: 911919.

Krogan, N.J., Kim, M., Ahn, S.H., Zhong, G., Kobor, M.S., Cagney, G., Emili, A., Shilatifard, A., Buratowski, S., and Greenblatt, J.F. 2002. RNA polymerase II elongation factors of Saccharomyces cerevisiae: A targeted proteomics approach. Mol. Cell. Biol. 22: 6979-6992.

Krogan, N.J., Dover, J., Wood, A., Schneider, J., Heidt, J., Boateng, M.A., Dean, K., Ryan, O.W., Golshani, A., Johnston, M., et al. 2003. The Paf1 complex is required for histone H3 methylation by COMPASS and Dotlp: Linking transcriptional elongation to histone methylation. Mol. Cell 11: 721-729.

Lee, I., Bleecker, A., and Amasino, R. 1993. Analysis of naturally occurring late flowering in Arabidopsis thaliana. Mol. Gen. Genet. 237: 171-176.

Lee, I., Michaels, S.D., Masshardt, A.S., and Amasino, R.M. 1994. The late-flowering phenotype of FRIGIDA and LUMINIDEPENDENS is suppressed in the Landsberg erecta strain of Arabidopsis. Plant I. 6: 903-909.

Michaels, S. and Amasino, R. 1999. FLOWERING LOCUS C encodes a novel MADS domain protein that acts as a repressor of flowering. Plant Cell 11: 949-956. 
2000. Memories of winter: Vernalization and the competence to flower. Plant Cell Environ. 23: 1145-1154.

- 2001. Loss of FLOWERING LOCUS C activity eliminates the late-flowering phenotype of FRIGIDA and autonomous pathway mutations but not responsiveness to vernalization. Plant Cell 13: 935-941.

Michaels, S.D., He, Y., Scortecci, K.C., and Amasino, R.M. 2003. Attenuation of FLOWERING LOCUS C activity as a mechanism for the evolution of summer-annual flowering behavior in Arabidopsis. Proc. Natl. Acad. Sci. 100: 1010210107.

Murtas, G., Reeves, P.H., Fu, Y.F., Bancroft, I., Dean, C., and Coupland, G. 2003. A nuclear protease required for flowering-time regulation in Arabidopsis reduces the abundance of SMALL UBIQUITIN-RELATED MODIFIER conjugates. Plant Cell 15: 2308-2319.

Ng, H.H., Robert, F., Young, R.A., and Struhl, K. 2003. Targeted recruitment of Set1 histone methylase by elongating Pol II provides a localized mark and memory of recent transcriptional activity. Mol. Cell 11: 709-719.

Noh, Y.S. and Amasino, R.M. 2003. PIE1, an ISWI family gene, is required for FLC activation and floral repression in Arabidopsis. Plant Cell 15: 1671-1682.

Parenicova, L., de Folter, S., Kieffer, M., Horner, D.S., Favalli, C., Busscher, J., Cook, H.E., Ingram, R.M., Kater, M.M., Davies, B., et al. 2003. Molecular and phylogenetic analyses of the complete MADS-box transcription factor family in Arabidopsis: New openings to the MADS world. Plant Cell 15: 1538-1551.

Putterill, J., Laurie, R., and Macknight, R. 2004. It's time to flower: The genetic control of flowering time. Bioessays 26: $363-373$.

Ratcliffe, O.J., Nadzan, G.C., Reuber, T.L., and Riechmann, J.L. 2001. Regulation of flowering in Arabidopsis by an FLC homologue. Plant Physiol. 126: 122-132.

Ratcliffe, O.J., Kumimoto, R.W., Wong, B.J., and Riechmann, J.L. 2003. Analysis of the Arabidopsis MADS AFFECTING FLOWERING gene family: MAF2 prevents vernalization by short periods of cold. Plant Cell 15: 1159-1169.

Reeves, P.H., Murtas, G., Dash, S., and Coupland, G. 2002. early in short days 4, a mutation in Arabidopsis that causes early flowering and reduces the mRNA abundance of the floral repressor FLC. Development 129: 5349-5361.

Roguev, A., Schaft, D., Shevchenko, A., Pijnappel, W.W., Wilm, M., Aasland, R., and Stewart, A.F. 2001. The Saccharomyces cerevisiae Set1 complex includes an Ash2 homologue and methylates histone 3 lysine 4. EMBO J. 20: 7137-7148.

Sanda, S.L. and Amasino, R.M. 1996. Ecotype-specific expression of a flowering mutant phenotype in Arabidopsis thaliana. Plant Physiol. 111: 641-645.

Santos-Rosa, H., Schneider, R., Bannister, A.J., Sherriff, J., Bernstein, B.E., Emre, N.C., Schreiber, S.L., Mellor, J., and Kouzarides, T. 2002. Active genes are tri-methylated at K4 of histone H3. Nature 419: 407-411.

Santos-Rosa, H., Schneider, R., Bernstein, B.E., Karabetsou, N., Morillon, A., Weise, C., Schreiber, S.L., Mellor, J., and Kouzarides, T. 2003. Methylation of histone H3 K4 mediates association of the Isw1p ATPase with chromatin. Mol. Cell 12: $1325-1332$.

Schomburg, F.M., Patton, D.A., Meinke, D.W., and Amasino, R.M. 2001. FPA, a gene involved in floral induction in Arabidopsis, encodes a protein containing RNA-recognition motifs. Plant Cell 13: 1427-1436.

Scortecci, K.C., Michaels, S.D., and Amasino, R.M. 2001. Identification of a MADS-box gene, FLOWERING LOCUS M, that represses flowering. Plant J. 26: 229-236.
- 2003. Genetic interactions between FLM and other flowering-time genes in Arabidopsis thaliana. Plant Mol. Biol. 52: 915-922.

Sheldon, C.C., Burn, J.E., Perez, P.P., Metzger, J., Edwards, J.A., Peacock, W.J., and Dennis, E.S. 1999. The FLF MADS box gene: a repressor of flowering in Arabidopsis regulated by vernalization and methylation. Plant Cell 11: 445-458.

Shi, X., Finkelstein, A., Wolf, A.J., Wade, P.A., Burton, Z.F., and Jaehning, J.A. 1996. Paflp, an RNA polymerase II-associated factor in Saccharomyces cerevisiae, may have both positive and negative roles in transcription. Mol. Cell. Biol. 16: 669676.

Soppe, W.J., Bentsink, L., and Koornneef, M. 1999. The earlyflowering mutant efs is involved in the autonomous promotion pathway of Arabidopsis thaliana. Development 126: 4763-4770.

Squazzo, S.L., Costa, P.J., Lindstrom, D.L., Kumer, K.E., Simic, R., Jennings, J.L., Link, A.J., Arndt, K.M., and Hartzog, G.A. 2002. The Paf1 complex physically and functionally associates with transcription elongation factors in vivo. EMBO $J$. 21: 1764-1774.

Sung, S. and Amasino, R.M. 2004. Vernalization in Arabidopsis thaliana is mediated by the PHD finger protein VIN3. Nature 427: 159-164.

Tadege, M., Sheldon, C.C., Helliwell, C.A., Stoutjesdijk, P., Dennis, E.S., and Peacock, W.J. 2001. Control of flowering time by FLC orthologues in Brassica napus. Plant J. 28: 545553.

Tsukiyama, T., Palmer, J., Landel, C.C., Shiloach, J., and Wu, C. 1999. Characterization of the imitation switch subfamily of ATP-dependent chromatin-remodeling factors in Saccharomyces cerevisiae. Genes \& Dev. 13: 686-697.

Zhang, H. and van Nocker, S. 2002. The VERNALIZATION INDEPENDENCE 4 gene encodes a novel regulator of FLOWERING LOCUS C. Plant J. 31: 663-673.

Zhang, H., Ransom, C., Ludwig, P., and van Nocker, S. 2003. Genetic analysis of early flowering mutants in Arabidopsis defines a class of pleiotropic developmental regulator required for expression of the flowering-time switch FLOWERING LOCUS C. Genetics 164: 347-358. 


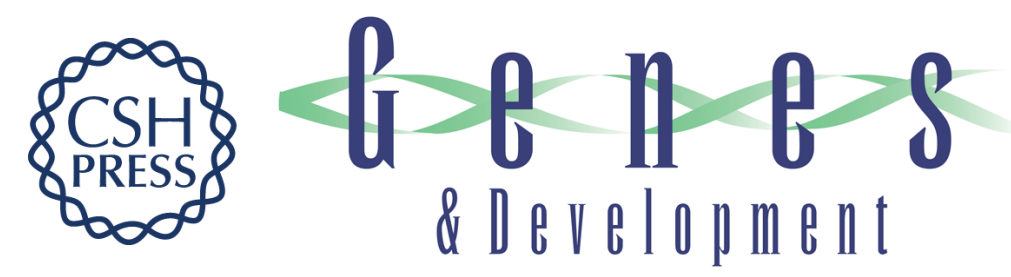

\section{PAF1-complex-mediated histone methylation of FLOWERING LOCUS C chromatin is required for the vernalization-responsive, winter-annual habit in Arabidopsis}

Yuehui He, Mark R. Doyle and Richard M. Amasino

Genes Dev. 2004, 18:

Access the most recent version at doi:10.1101/gad.1244504

Supplemental http://genesdev.cshlp.org/content/suppl/2004/11/08/gad.1244504.DC1
Material

References This article cites 49 articles, 26 of which can be accessed free at: http://genesdev.cshlp.org/content/18/22/2774.full.html\#ref-list-1

License

Email Alerting Receive free email alerts when new articles cite this article - sign up in the box at the Service top right corner of the article or click here.

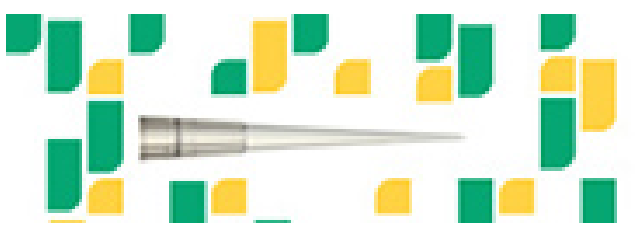

Focused on your science. 